PROCEEDINGS OF THE

AMERICAN MATHEMATICAL SOCIETY

Volume 130, Number 9, Pages 2535-2541

S 0002-9939(02)06478-X

Article electronically published on April 10, 2002

\title{
A NOTE ON THE DEFINING EQUATIONS OF SINGULAR VARIETIES
}

\author{
SEUNGHUN LEE
}

(Communicated by Michael Stillman)

\begin{abstract}
We show that the vanishing theorem of Bertram, Ein, and Lazarsfeld also holds for varieties with mild singularities.
\end{abstract}

\section{INTRODUCTION}

In this paper, we show that the following vanishing theorem of Bertram, Ein, and Lazarsfeld also holds for varieties with mild singularities.

Theorem 0.1 (Theorem 7 in [3]). Let $X$ be a smooth projective variety and let $Z$ be a smooth subvariety of $X$. Let $A, H$ be Cartier divisors on $X$ such that $|A|$ is base point free and $H$ is ample. Assume that $Z$ is cut out scheme-theoretically by $m$ sections $s_{i}$ of $H^{0}\left(X, \mathcal{O}_{X}\left(d_{i} A\right)\right)$, where $d_{1} \geq d_{2} \geq \cdots \geq d_{m}$ for some $m$. Then

$$
H^{k}\left(X, \mathcal{I}_{Z}^{a} \otimes \mathcal{O}_{X}\left(K_{X}+t A+H\right)\right)=0
$$

for all $k, a>0$ and $t \geq a d_{1}+d_{2}+\cdots+d_{e}$, where $e=\operatorname{codim}_{X} Z$.

Using this theorem and its variations, they obtained a number of interesting results on the defining equations of smooth projective varieties. We refer to 3 for the details. Meanwhile, in 2 2 Bertram suggested a new approach using multiplier ideals technique. Here we follow his argument to improve their theorem. The following is our main result.

Theorem 0.2. Let $X$ be a projective variety with log terminal singularities and let $Z$ be a subvariety of $X$ such that $X$ is smooth at the generic point of $Z$. Let $A$ be a Cartier divisor on $X$ such that $|A|$ is base point free and $Z$ is cut out schemetheoretically by $m$ sections $s_{i}$ of $H^{0}\left(X, \mathcal{O}_{X}\left(d_{i} A\right)\right)$, where $d_{1} \geq d_{2} \geq \cdots \geq d_{m}$ for some $m$. Let $H$ be a Cartier divisor on $X$ such that $H-K_{X}-\left(a d_{1}+d_{2}+\cdots+d_{e}\right) A$ is ample. If $Z$ is log terminal and the ideal sheaf $\mathcal{I}_{Z / X}$ is locally generated by $\mathcal{O}_{X}-$ regular sequences, then

$$
H^{k}\left(X, \mathcal{I}_{Z}^{a} \otimes \mathcal{O}_{X}(H)\right)=0
$$

for all $k, a>0$, where $e=\operatorname{codim}_{X} Z$.

Received by the editors January 17, 2000 and, in revised form, April 10, 2001.

2000 Mathematics Subject Classification. Primary 14F17; Secondary 14C20.

The work was supported by grant No. R01-1999-00004 from the Korea Science and Engineering Foundation. 
As in [3], we have the following corollaries.

Corollary 0.3. Let $Z \subset \mathbb{P}^{n}$ be a projective variety of codimension e, cut out scheme-theoretically by hypersurfaces of degree $d_{1} \geq \cdots \geq d_{m}$ for some $m$. Assume that $Z$ has only log terminal singularities and $\mathcal{I}_{Z / \mathbb{P}^{n}}$ is locally generated by $\mathcal{O}_{X}$-regular sequences. Then

$$
H^{k}\left(\mathbb{P}^{n}, \mathcal{I}_{Z}^{a} \otimes \mathcal{O}_{\mathbb{P}^{n}}(t)\right)=0
$$

for all $k, a>0$, and $t \geq-n+a d_{1}+d_{2}+\cdots+d_{e}$.

Corollary 0.4. Let $Z \subset \mathbb{P}^{n}$ be a projective variety of codimension e and degree $d$. Assume that $Z$ has only log terminal singularities and $\mathcal{I}_{Z / \mathbb{P}^{n}}$ is locally generated by $\mathcal{O}_{X}$-regular sequences. Then

$$
H^{k}\left(\mathbb{P}^{n}, \mathcal{I}_{Z}^{a} \otimes \mathcal{O}_{\mathbb{P}^{n}}(t)\right)=0
$$

for all $k, a>0$, and $t \geq-n+(a-1) d+e d$.

The new ingredient in the paper is the connectedness theorem of Shokurov, Theorem 1.6. It is used to make sure that certain pairs are log canonical. In the statement of the previous results, since the ideal sheaf is generated by a regular sequence, $X$ is already canonical. But it is expected to be true without the regularity condition. So we prefer to state it this way in a hope that we can remove the condition in the future. In this note we work over the field of complex numbers.

I would like to thank Prof. Lawrence Ein for directing my attention to this problem, and sharing with me many stimulating conversations. I would also like to thank Prof. Fujita, Prof. Helmke and Prof. Kawamata for their helpful comments.

\section{Preliminary}

Here we collect known results which are used in the proof.

Definition 1.1. Let $Z$ be a subvariety of a variety $X$. Let $L_{i}, i=1, \ldots, m$, be line bundles on $X$. We say that $Z$ is cut out scheme-theoretically by $m$ sections $s_{i}$ in $H^{0}\left(X, L_{i}\right)$ if each $s_{i}$ vanishes at $Z$ and $\bigoplus L_{i}^{-1} \longrightarrow \mathcal{I}_{Z} \rightarrow 0$.

Definition 1.2. (1) Let $X$ be a variety. Let $D$ be a $Q$-Weil divisor in $X$. Let $D=\sum d_{i} D_{i}$, where $D_{i}$ 's are prime divisors. Then we write $\lceil D\rceil:=\sum\left\lceil d_{i}\right\rceil D_{i}$, where $\lceil d\rceil$ is the smallest integer greater than or equal to $d$.

(2) Let $X$ be a normal variety. Let $\Delta$ be an effective $Q$-divisor on $X$ such that $K_{X}+\Delta$ is $Q$-Cartier. Let $f: Y \rightarrow X$ be an embedded resolution of $(X, \Delta)$. Let

$$
K_{Y}+f_{*}^{-1} \Delta=f^{*}\left(K_{X}+\Delta\right)+\sum a_{i} F_{i},
$$

where the $F_{i}$ 's are exceptional divisors and $a_{i} \in \mathbb{Q}$. We say that $(X, \Delta)$ is

$$
\begin{array}{ll}
\text { canonical } & \text { if } a_{i} \geq 0, \\
\text { lt (or log terminal) } & \text { if } a_{i}>-1 \text { and } \Delta=0, \\
\text { klt (or Kawamata log terminal) } & \text { if } a_{i}>-1 \text { and }\lceil-\Delta\rceil=0, \\
\text { plt (or purely log terminal) } & \text { if } a_{i}>-1, \\
\text { lc (or log canonical) } & \text { if } a_{i} \geq-1 .
\end{array}
$$


We refer to [7] and [8] for the proof of the following theorems.

Theorem 1.3. Let $(X, \Delta)$ be a Kawamata log terminal pair. Let $f: X \rightarrow S$ be a proper morphism onto a variety $S$. Let $D$ be a $Q$-Cartier Weil divisor on $X$ such that $D-K_{X}-\Delta$ is f-nef and f-big. Then $R^{i} f_{*} \mathcal{O}_{X}(D)=0$ for $i>0$.

Theorem 1.4. Let $X$ be a normal variety and let $\Lambda$ be a (not necessary complete) linear system of Weil divisors.

(1) Let $(X, \Delta)$ be a log canonical pair. Then $(X, \Delta+D)$ is also log canonical for generic $D \in \Lambda$.

(2) Let $(X, \Delta)$ be a Kawamata log terminal pair. Then $(X, \Delta+b D)$ is also Kawamata log terminal for generic $D \in \Lambda$ and $0 \leq b<1$.

Theorem 1.5. Let $X$ be a normal variety and let $S \subset X$ be a normal irreducible Cartier divisor. Let $B$ an effective $Q$-divisor such that $K_{X}+S+B$ is $Q$-Cartier.

(1) $(X, S+B)$ is plt near $S$ if and only if $\left(S,\left.B\right|_{S}\right)$ is klt.

(2) Assume that $B \neq \emptyset$ and $S$ is log terminal. Then $(X, S+B)$ is lc near $S$ if and only if $\left(S,\left.B\right|_{S}\right)$ is lc.

Multiplier ideals. Here we briefly recall the multiplier ideals and list two of their properties. For the details, we refer to [4. There things are defined on smooth settings. But with minor modifications, the definition can be extended to the logterminal setting.

Let $X$ be a $\log$ terminal variety, let $G$ be an effective $Q$-Cartier divisor, and let $f: Y \longrightarrow X$ be the embedded resolution of $G$ such that the support, $\sum F_{i}$, of $f^{*} G$ and $f$-exceptional divisors is in simple normal crossing. Let $K_{Y}-f^{*} K_{X}=\sum b_{i} F_{i}$ and $f^{*} G=\sum g_{i} F_{i}$. Let $\sum\left\lceil\left(b_{i}-g_{i}\right)\right\rceil F_{i}=P-N$, where $P$ and $N$ are effective divisors with no common components. From Lemma 1.3.2 in [7],

$$
f_{*} \mathcal{O}_{Y}(P-N)=f_{*} \mathcal{O}_{Y}(-N) \subset \mathcal{O}_{X}
$$

We call $f_{*} \mathcal{O}_{Y}(P-N)$ the multiplier ideal for $G$. Let $Z(G)$ be the corresponding scheme, and denote the multiplier ideal for $G$ by $\mathcal{I}_{Z(G)}$. Using a fairly standard argument, one can show that $\mathcal{I}_{Z(G)}$ does not depend on the choice of the resolution.

Theorem 1.6. In the above notation, $N \cap f^{-1}(p)$ is connected for all closed points $p \in Z(G)$.

Theorem 1.7. Let $X$ and $G$ be as above. Let $H$ be a Cartier divisor on $X$ such that $H-K_{X}-G$ is nef and big. Then $H^{k}\left(X, \mathcal{I}_{Z(G)} \otimes \mathcal{O}_{X}(H)\right)=0$ for all $k>0$.

\section{Proof of Theorem 0.2}

We set $A_{i}:=d_{i} A$. Let $f: \bar{X} \longrightarrow X$ be the blowing up at $Z$ with the exceptional divisor $E$ and let $g: Y \longrightarrow \bar{X}$ be an embedded resolution of $E$. We set $h=f \circ g$, and $F=g^{*} E$. Now choose a generic divisor

$$
B_{i} \in\left|\mathcal{O}_{X}\left(A_{i}\right) \otimes \mathcal{I}_{Z}\right|
$$

for $i=1, \cdots, m$, and let $f^{*} B_{i}=\bar{B}_{i}+E, D_{i}=g^{*} \bar{B}_{i}$. We set $X_{0}=X, \bar{X}_{0}=\bar{X}$, $Y_{0}=Y, X_{i}=\bigcap_{j=1}^{i} B_{j}, \bar{X}_{i}=\bigcap_{j=1}^{i} \bar{B}_{j}$, and $Y_{i}=\bigcap_{j=1}^{i} D_{j}$.

Lemma 2.1 (Claim 1.5 in [3]). With the same notation,

(1) $(\bar{X}, E)$ is purely log terminal and $K_{\bar{X}} \underset{\text { lin }}{\stackrel{Q}{2}} f^{*} K_{X}+(e-1) E$.

(2) $\bigcap_{k=1}^{i-1} B_{k} \cap B s\left|f^{*} A_{i}-E\right|=\emptyset$ for all $i=1, \cdots, m$. 
Proof. (1) Since the ideal sheaf of $Z$ is generated by a regular sequence, $E$ is irreducible and $\log$ terminal. Let

$$
U=\bar{X}-f^{-1}(\operatorname{Sing} X \cup \operatorname{Sing} Z) .
$$

Then, since $X$ is smooth at generic points of $Z, \operatorname{codim} \bar{X}-U \geq 2$. Thus $\bar{X}$ is smooth in codimension 1. Now for a moment we assume that $\bar{X}$ is CohenMacaulay, so that $\bar{X}$ is normal. Let $i$ be the integer such that $i K_{X}$ is Cartier. Then $\mathcal{O}_{U}\left(i K_{\bar{X}}\right)=\mathcal{O}_{U}\left(i f^{*} K_{X}+i(e-1) E\right)$. Since $\mathcal{O}_{\bar{X}}\left(i K_{\bar{X}}\right)$ is a divisorial sheaf and $\bar{X}$ is normal (cf. [9]),

$$
\mathcal{O}_{\bar{X}}\left(i K_{\bar{X}}\right)=\mathcal{O}_{\bar{X}}\left(i f^{*} K_{X}+i(e-1) E\right) .
$$

Now $(\bar{X}, E)$ is purely log terminal by Theorem 1.5.1.

To show that $\bar{X}$ is Cohen-Macaulay, we may assume that $X=\operatorname{Spec}(R)$ for a Noetherian local $\operatorname{ring} R$ with the maximal ideal $m$, and $Z=\operatorname{Spec}(R / I)$, where $I$ is an ideal of $R$ generated by an $R$-regular sequence $\left(a_{1}, \cdots, a_{e}\right)$ in $m$. We induct on the codimension of $Z$. If $e=1$, then $Z$ is a Cartier divisor in $X$. Thus $\bar{X}=X$ and $\bar{X}$ is Cohen-Macaulay. Now assume that $e>1$. We choose a close point $p$ in $E$. Then there are constants $\lambda_{1}, \cdots, \lambda_{e}$ in $\mathbb{C}$ such that the proper transform of $V\left(\lambda_{1} a_{1}+\cdots+\lambda_{e} a_{e}\right)$ in $\bar{X}$ contains $p$. We put

$$
a_{e}^{\prime}=\lambda_{1} a_{1}+\cdots+\lambda_{e} a_{e} .
$$

Since being a regular sequence does not depend on the order of the elements in the sequence, we may assume that $\lambda_{e} \neq 0$. Suppose there is $b \in A$ such that $b \cdot a_{e}^{\prime} \in\left(a_{1}, \cdots, a_{e-1}\right)$. Then $b \cdot \lambda_{e} a_{e} \in\left(a_{1}, \cdots, a_{e-1}\right)$ and $b \in\left(a_{1}, \cdots, a_{e-1}\right)$. Thus $\left(a_{1}, \cdots, a_{e-1}, a_{e}^{\prime}\right)$ is also an $A$-regular sequence in $m$. Now let $V=\operatorname{Spec}\left(R /\left(a_{e}^{\prime}\right)\right)$. Since $X$ is Cohen-Macaulay, $V$ is also Cohen-Macaulay. Then from the induction hypothesis, the blowing up of $V$ at $\mathcal{I}_{Z / V}$ is also Cohen-Macaulay. Thus $\bar{X}$ is CohenMacaulay at $p$.

(2) Let $F_{i} \in\left|f^{*} A_{i}-E\right|$ be the divisor corresponding to $s_{i}$. Since $Z$ is cut out scheme-theoretically, $\bigoplus_{i=1}^{m} \mathcal{O}_{\bar{X}}\left(-f^{*} A_{i}+E\right) \longrightarrow \mathcal{O}_{\bar{X}} \longrightarrow 0$. Thus $\bigcap_{i=1}^{m} F_{i}=\emptyset$. Now $B_{i}$ being generic implies that

$$
\bigcap_{k=1}^{i-1} B_{k} \cap \bigcap_{k=i}^{m} F_{k}=\emptyset .
$$

Since $\left|A_{i}-A_{j}\right|$ is base point free for all $j \geq i$,

$$
B s\left|f^{*} A_{i}-E\right| \subset \bigcap_{j=i}^{m} B s\left|f^{*} A_{j}-E\right| \subset \bigcap_{j=i}^{m} F_{j}
$$

for all $i \geq 1$. Thus $\bigcap_{k=1}^{i-1} B_{k} \cap B s\left|f^{*} A_{i}-E\right|=\emptyset$.

The following lemma is due to Bertram. We give the proof for the convenience of the readers.

Lemma 2.2 (Proposition 2.1 in [2]). If $\left(X, B_{1}+\cdots+B_{e}\right)$ is log canonical, then $H^{k}\left(X, \mathcal{I}_{Z}^{a} \otimes \mathcal{O}_{X}(H)\right)=0$ for all $k, a>0$.

Proof. First we note that $\mathcal{I}_{Z\left(a B_{1}+B_{2}+\cdots+B_{e}\right)}$ and $\mathcal{I}_{Z}^{a}$ agree at the generic point of $Z$. Now from the assumption and $H-K_{X}-\left(a d_{1}+d_{2}+\cdots+d_{e}\right) A$ being ample, we can perturb $a B_{1}+B_{2}+\cdots+B_{e}$ a little bit to obtain an effective $Q$-Cartier divisor $G$ such that 
(1) $H-K_{X}-G$ is ample, and

(2) $\mathcal{I}_{Z(G)}=\mathcal{I}_{Z}^{a} \otimes \mathcal{I}_{W}$, where $W \cap Z=\emptyset$.

Then, from (1) and Theorem 1.7, $H^{k}\left(X, \mathcal{I}_{Z(G)} \otimes \mathcal{O}_{X}(H)\right)=0$ for all $k>0$. Consider the following exact sequence:

$$
0 \longrightarrow \mathcal{I}_{Z(G)} \otimes \mathcal{O}_{X}(H) \longrightarrow \mathcal{O}_{X}(H) \longrightarrow \mathcal{O}_{Z(G)}(H) \longrightarrow 0 .
$$

From Theorem 1.3, $H^{k}\left(X, \mathcal{O}_{X}(H)\right)=0$ for all $k>0$. Thus

$$
H^{k}\left(Z(G), \mathcal{O}_{Z(G)}(H)\right)=0
$$

for all $k>0$. So by $(2), H^{k}\left(W, \mathcal{O}_{W}(H)\right)=0$ for all $k>0$. Now the vanishing follows from the exact sequence

$$
0 \longrightarrow \mathcal{I}_{Z(G)} \otimes \mathcal{O}_{X}(H) \longrightarrow \mathcal{I}_{Z}^{a} \otimes \mathcal{O}_{X}(H) \longrightarrow \mathcal{O}_{W}(H) \longrightarrow 0 .
$$

To show that $\left(X, B_{1}+\cdots+B_{e}\right)$ is log canonical, we will use the following lemma to reduce to the codimension 2 case.

Lemma 2.3. We fix $1 \leq i \leq e-1$ and let $B$ be the unique irreducible component of $\left.B_{i}\right|_{X_{i-1}}$ containing $Z$. We denote by $\bar{B}$ and $D$ the proper transform of $B$ in $\bar{X}_{i-1}$ and in $Y_{i-1}$ respectively. Then:

(1) $\left.B_{i}\right|_{X_{i-1}}=B$ near $Z$, and $E \cap \bar{B}$ is irreducible.

(2) $B$ is log terminal near $Z$.

Proof. (1) We use induction. We assume that it is true for $j:=i-1$. Let $f_{j}=\left.f\right|_{\bar{X}_{j}}$ and $E_{j}=E \cap \bar{X}_{j}$. Since $E_{j}$ is irreducible,

$$
\operatorname{dim} f_{j}^{-1}(W) \leq \operatorname{dim} X_{j}-2,
$$

where $W=\operatorname{Sing} Z \cup\left\{Z \cap \operatorname{Sing} X_{j}\right\}$. Since $B_{i}$ is generic, from the above inequality and Lemma 2.1.2, every irreducible component of $\left.\bar{B}_{i}\right|_{\bar{X}_{j}}$ intersecting $E_{j}$ also intersects $E_{j}-f_{j}^{-1}(W)$. Thus for dimension reasons, for such components except $\bar{B}$, they should contains a whole fiber of $\left.f_{j}\right|_{E_{j}}$. So they must intersect with $\bar{B}$, which contradicts Lemma 2.1.2 and Bertini's theorem. Thus $\bar{B}$ is the unique component intersecting $E_{j}$.

Now to show that $E_{j} \cap \bar{B}$ is irreducible, again we note that

$$
\operatorname{dim} f_{j}^{-1}(W) \leq \operatorname{dim} E_{j}-1 .
$$

If $E_{j} \cap \bar{B}$ is not irreducible, then every component of $E_{j} \cap \bar{B}$ except the one that dominates $Z$ must lie in $f_{j}^{-1}(W)$. These components cannot move, which contradicts Lemma 2.1.2.

(2) Since $X$ is Cohen-Macaulay, $B$ is also Cohen-Macaulay near $Z$ by the previous result. Thus from Lemma $2.1 .2, B$ is normal near $Z$. Since $\left\lceil\left.\left(K_{Y / X}+(e-1-i) F\right)\right|_{D}\right\rceil$ is effective by Lemmas 2.1.1 and 2.1.2 in an open neighborhood over $Z$, it follows that $\left\lceil K_{Y}+D_{1}+\cdots+D_{i}-\left.h^{*}\left(K_{X}+B_{1}+\cdots+B_{i}\right)\right|_{D}\right\rceil$ is also effective. Thus $B$ is $\log$ terminal near $Z$.

Note that it is now enough to show that $\left(X_{e-2},\left.B_{e-1}\right|_{X_{e-2}}+\left.B_{e}\right|_{X_{e-2}}\right)$ is $\log$ canonical near $Z$. In fact, using Theorem 1.5.2 and Lemma 2.3.2 repeatedly, we obtain that $\left(X, B_{1}+\cdots+B_{e}\right)$ is $\log$ canonical near $Z$. We set

$$
\Delta_{\epsilon}=\left.\left(\bar{B}_{e-1}+(1-\epsilon) \bar{B}_{e}+(1-\epsilon) E\right)\right|_{\bar{X}_{e-2}} .
$$


Since $(\bar{X}, E)$ is purely $\log$ terminal by Lemma 2.1.1, from Theorem 1.4.1 and Lemma 2.1.2, $\left(\bar{X}_{e-2}, \Delta_{0}\right)$ is $\log$ canonical on an open set containing $\left.\bar{B}_{e-1}\right|_{\bar{X}_{e-2}} \cap E$. Now suppose that $\left(\bar{X}_{e-2}, \Delta_{0}\right)$ is not $\log$ canonical at $y \in E_{x}=\left.f^{-1}(x) \cap E\right|_{\bar{X}_{e-2}}$ for some $x \in Z$. Then for $0<\epsilon<<1,\left(\bar{X}_{e-2}, \Delta_{\epsilon}\right)$ is not $\log$ canonical at $y$. Thus from Theorem 1.6, $\left(\bar{X}_{e-2}, \Delta_{\epsilon}\right)$ is not Kawamata log terminal along $E_{x}$. However $\left.\bar{B}_{e-1}\right|_{\bar{X}_{e-2}}$ has no components containing $E_{x}$. Thus

$$
\left(\bar{X}_{e-2},\left.(1-\epsilon) \bar{B}_{e-1}\right|_{\bar{X}_{e-2}}+\left.(1-\epsilon) \bar{B}_{e}\right|_{\bar{X}_{e-2}}+\left.(1-\epsilon) E\right|_{\bar{X}_{e-2}}\right)
$$

is not Kawamata log terminal along $E_{x}$, especially at $\left.E_{x} \cap \bar{B}_{e-1}\right|_{\bar{X}_{e-2}}$. This contradicts Theorem 1.4.2.

\section{REMARKS}

Here we make some remarks on the proof and the condition imposed on $Z$ in Theorem 0.2. We keep the same notation as in $\S 2$.

3.1. Log terminal singularities. Log terminal singularities are normal by definition. Thus on curves they are simply smooth points. For surfaces or higher dimensional varieties, it is well-known that quotient singularities are log terminal, and log terminal singularities are rational (cf. [5], 7], 9]). So, Du Val singularities (rational double points),

$$
\begin{aligned}
& A_{n}: x^{2}+y^{2}+z^{n+1} \text { for } n \geq 1, \\
& D_{n}: x^{2}+y^{2} z+z^{n-1} \text { for } n \geq 4, \\
& E_{6}: x^{2}+y^{3}+z^{4}, \\
& E_{7}: x^{2}+y^{3}+y z^{3}, \\
& E_{8}: x^{2}+y^{3}+z^{5},
\end{aligned}
$$

are log terminal, where $x, y, z$ is an analytic coordinate of $\mathbb{C}^{3}$ at $(0,0,0)$. And the threefold hypersurface singularities given locally analytically by the following type are known [9, 2.1] to be log terminal at $(0,0,0,0)$ :

$$
f(x, y, z)+t g(x, y, z, t)=0,
$$

where $f(x, y, z)$ is one of the five polynomials above and $g(x, y, z, t)$ is arbitrary. For example,

$$
S: \sum_{i=0}^{3} \frac{x_{0} x_{1} x_{2} x_{3}}{x_{i}}=0
$$

has four $A_{1}$-type singularities at $(1,0,0,0),(0,1,0,0),(0,0,1,0)$, and $(0,0,0,1)$. Thus Theorem 0.2 applies to $S$ and its Veronese imbeddings. On surfaces, however, log terminal singularities are also quotient singularities. But there are rational surface singularities that are not log terminal. Kawamata [6] has shown that minimal centers ( $Z$ in our proof is one of them) has only log terminal singularities.

3.2. $Z$ with isolated singularities. As Ein has kindly pointed out to us, if $Z$ has only isolated singularities, then we get the same vanishing theorem. In fact, a similar argument would produce an ideal sheaf $\mathcal{J}$ so that $0 \rightarrow \mathcal{J} \rightarrow \mathcal{I}_{Z}^{a} \longrightarrow Q \rightarrow 0$ is exact, $\operatorname{dim} \operatorname{Supp} Q=0$, and the vanishing in Theorem 0.2 holds for $\mathcal{J}$. Thus $\mathcal{I}_{Z}^{a}$ also has the vanishing properties. As a corollary, we see that the main theorem also applies to $Z$ if $Z$ is a normal surface. 
3.3. $Z$ with $d_{1}=\cdots=d_{e}$. As observed in 3], if $d_{1}=\cdots=d_{e}$, as in Corollary 3, then we can weaken the assumption on $Z$ of Theorem 0.2 considerably. In fact, it is enough to assume that

$$
h_{*} \mathcal{O}_{Y}\left(\left\lceil K_{Y / X}-(e+a-1) F\right\rceil\right)=\mathcal{I}_{Z}^{a} .
$$

This is true if, for example, $E$ is irreducible. It would be interesting to know if $Z$ being log terminal implies (3.1). For surfaces, it is easy to prove.

Proposition 3.1. Let $p \in S \subset \mathbb{C}^{n}$ be a log terminal singularity. Then the exceptional divisor of the blowing up of $\mathbb{C}^{n}$ at $\mathcal{I}_{S}$ is irreducible.

Proof. Log terminal surface singularities are rational surface singularities (cf. [5]). Thus the multiplicity $m$ of $S$ at $p$ is one less than the embedded dimension of $S$ at $p$ [1]. So we may assume that $n=m+1$. Now we cut out $S$ by two generic hyperplanes through $p$ to obtain a zero dimensional subscheme $W \subset \mathbb{C}^{m-1}$. Since the length of $W$ is $m$ and the embedded dimension of $W$ is $m-1$, the ideal of $W$ in $\mathbb{C}^{m-1}$ is given by the square of the maximal ideal of $p$ in $\mathbb{C}^{m-1}$. But the blowing up of $\mathbb{C}^{m-1}$ at such an ideal is precisely the blowing up at the maximal ideal itself. Thus the exceptional divisor of the blowing up of $\mathbb{C}^{n}$ at $\mathcal{I}_{S}$ is irreducible.

\section{ACKNOWLEDGMENT}

This paper was written while the author was visiting Max-Planck-Institut für Mathematik at Bonn. I would like to thank the institute for their hospitality.

\section{REFERENCES}

1. M. Artin, On isolated rational singularities of surfaces, Amer. J. Math., 88 (1966), pp. 129136. MR 33:7340

2. A. Bertram An application of a log version of the Kodaira vanishing theorem to embedded projective varieties, alg-geom/9707001.

3. A. Bertram, L. Ein and R. Lazarsfeld Vanishing theorems, a theorem of Severi, and the equations defining projective varieties, J.Amer.Math.Soc., 4 (1991), pp. 587-602. MR 92g:14014

4. L. Ein, Multiplier ideals, Vanishing theorem and Applications Proc. Sympos. Pure. Math. Part1. 62, Amer. Math. Soc., Providence, RI, (1997) pp.203-219. MR 98m:14006

5. Y. Kawamata Crepant blowing-up of 3-dimensional canonical singularities and its application to degenerations of surfaces, Ann. of Math., 127 (1988), pp. 93-163. MR 89d:14023

6. Y. Kawamata Subadjunction of log canonical divisors II., Amer. J. Math., 120 (1998), pp. 893-899. MR 2000d:14020

7. Y. Kawamata, K. Matsuda, K. Matsuki Introduction to the minimal model problem Adv. Stud. Pure. Math., 10, North-Holland, Amsterdam-New York, (1987) pp.283-360. MR 89e:14015

8. J. Kollár Singularities of Pairs Proc. Sympos. Pure. Math. Part1. 62, Amer. Math. Soc., Providence, RI, (1997) pp.221-287. MR 99m:14033

9. M. Reid Canonical 3-folds Journées de Géometrie Algébique d'Angers, Sijthoff and Noordhoff, Alphen aan den Rijn, (1980) pp. 273-310. MR 82i:14025

Max-Planck-Institut für Mathematik Vivatsgasse 7, D-53111 Bonn, Germany Current address: Department of Mathematics, Konkuk University, Kwangjin-Gu Hwayangdong 1, Seoul 143-701, Korea

E-mail address: mbrs@kkucc.konkuk.ac.kr 